\title{
A review of interstellar polarization properties and recent measurements toward the Chamaeleon I and Taurus dark clouds
}

\author{
P. A. Gerakines ${ }^{1,2}$ and D. C. B. Whittet ${ }^{2}$ \\ ${ }^{1}$ Leiden Observatory, P.O. Box 9513, 2300 RA Leiden, The Netherlands \\ 2 Physics Department, Rensselaer Polytechnic Institute, Troy, NY 12180, U.S.A. \\ Received 10 October 1994; revised and accepted 20 January 1995
}

\begin{abstract}
The observed linear polarization of starlight is thought to be caused by magnetically aligned, elongated dust grains existing within interstellar clouds. Typically, the magnitude of polarization as a function of wavelength may be empirically fit with great accuracy by the Serkowski law, and comparisons of the parameters of this fit to extinction data provide information about grain sizes and environments. The linear polarization properties of the Chamaeleon I and Taurus dark clouds have been studied, using data from the literature combined with some new observations. Strong trends in the amount of polarization as a function of visual extinction $\left(P \vee v s, A_{V}\right)$ in the line of sight have been observed for both clouds. Specifically, the degree of polarization per unit extinction, or polarization efficiency $P / A_{\nu}$, for Taurus has been found to decrease dramatically with increasing $A_{v}$, the data being well fit by a power law. For Chamacleon, the relationship between the wavelength of maximum polarization $\left(\lambda_{\max }\right)$ and the ratio of total to selective extinction $\left(R_{V}\right)$ is significantly different from that shown previously for a variety of sources by Clayton and Mathis. It is suggested that these effects may be caused by the physical and chemical processes occurring in these clouds and may be used to further understand not only the grain aligmment mechanisms but also the environment of dense interstellar clouds.
\end{abstract}

\section{Introduction}

Linear polarization of starlight is seen towards many lines of sight where there exists extinction and is attributed to aligned, elongated dust grains. Data sets containing many lines of sight show clear correlations with the amount of

Correspondence to : P. A. Gerakines obscuring material. The first viable mechanism for producing interstellar linear polarization of starlight was put forward by Davis and Greenstein (DG) (1951). The DG mechanism achieves alignment through paramagnetic relaxation of thermally rotating grains, aligned perpendicular to some external magnetic field. Alignment is ultimately achieved by the internal torques which result from the application of this field on the spinning grain material [for a more complete discussion of the DG and other grain alignment mechanisms, see Whittet (1992), Chapter 4].

The DG mechanism explains the geometrical tendencies of interstellar polarization very accurately, but the high values of polarization observed imply degrees of alignment which are unattainable by this mechanism, given the observed value of the galactic magnetic field of a few $\mu$ Gauss [e.g. Heiles (1987)]. In order to account for this discrepancy, Jones and Spitzer (1967) proposed that the inclusion of ferromagnetic material within the grains could increase their magnetic susceptibility by a factor of $\sim 10^{6}$ over ordinary paramagnetic materials, leading to very efficient grain alignment. Furthermore, Mathis (1986) has developed a model in which a silicate grain is aligned if, and only if, it contains at least one such inclusion.

A second method of producing efficient grain alignment was proposed by Purcell (1979), called suprathermal spin. The energy released through the formation of molecules (such as molecular hydrogen) on grain surfaces will impart angular momentum to the grain. If, as discussed by Hollenbach and Salpeter (1971), $\mathrm{H}_{2}$ formation on grains occurs at preferential sites, then the distribution of these sites on the grain surface will affect the spin properties of the grain. A series of recombination events occurring at preferred sites on the grain surface could lead to grain angular velocities which are factors of $10^{4}-10^{5}$ above the expected values based on typical cloud conditions, increasing the efficiency to which grains may be aligned within interstellar clouds. 


\section{Chamaeleon I-Correlation of $\lambda_{\max }$ with $R_{\mathrm{R}}$}

The spectral dependence of visual and near-IR observations of polarization may be extremely well fit by the empirical Serkowski formula :

$$
p / p_{\max }=\exp \left[-K \ln ^{2}\left(\lambda_{\max } / \lambda\right)\right]
$$

(Serkowski, 1973; Coyne et al., 1974; Serkowski et al., 1975), where $p_{\max }$ is the peak percentage polarization and $\lambda_{\max }$ is the wavelength corresponding to $p_{\max }$.

$\lambda_{\max }$ and $R_{V}$ (the ratio of total to selective extinction, $A_{V}\left(E_{B-V}\right)$ are parameters which are proportional to the mean size of the particles responsible for polarization and extinction, respectively, at visible wavelengths. Mie theory calculations for dielectric cylinders show that polarization is most efficient when the quantity $2 \pi a(n-1) / \lambda$ is close to unity, where $a$ and $n$ are the radius and refractive index of the cylinder, and hence $\lambda_{\max } \simeq 2 \pi a(n-1)$. So, if the grains producing polarization also contribute significantly to visual extinction, or respond similarly to environmental influences, then a correlation should be expected between $\lambda_{\max }$ and $R_{V}$. Relations of this sort have been observed previously by various authors for sets which combine both diffuse and dark cloud environments (Serkowski et al., 1975 ; Whittet and van Breda, 1978 ; Clayton and Mathis, 1988).

In Fig. 1 [from Whittet et al.(1994)], we present a plot of $\lambda_{\max }$ vs $R_{V}$ for 38 stars in Chamaeleon I (Cha I), those

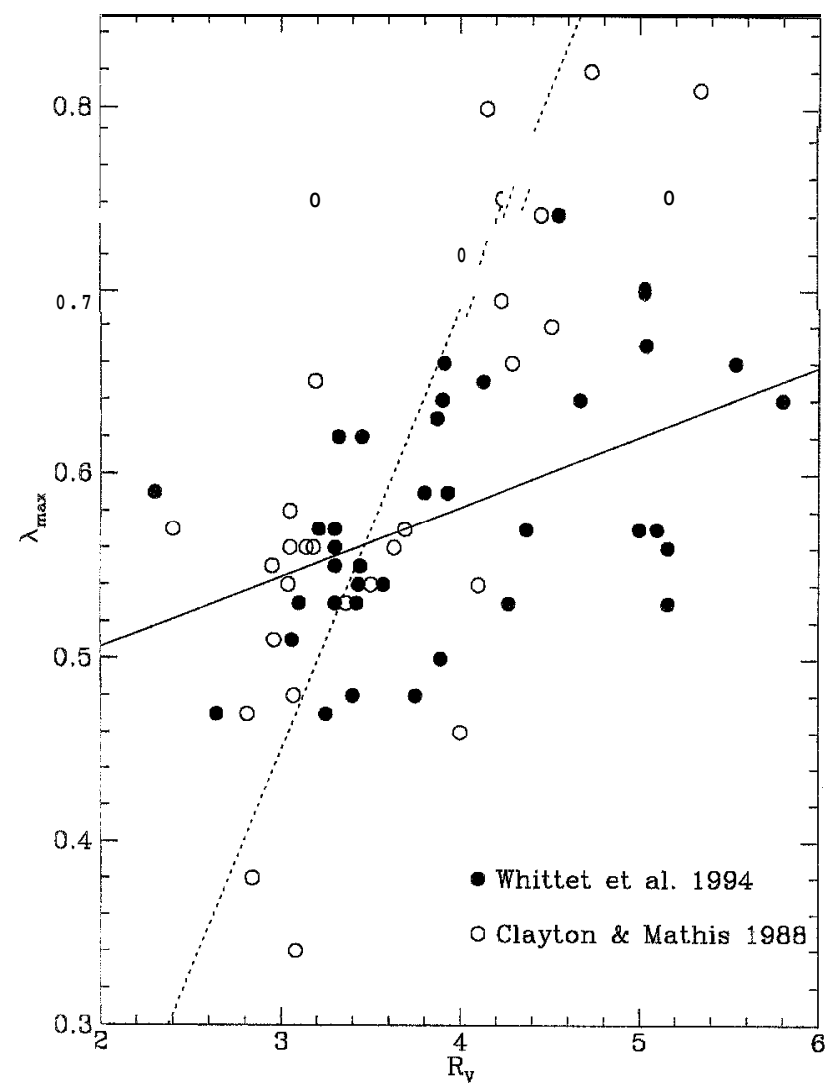

Fig. 1. Plot of $\lambda_{\max }$ vs $R_{V}$ for 38 stars in Cha I (filled circles) compared with 30 stars from Clayton and Mathis (CM; open circles). The solid and dotted lines represent least-squares fits to the Cha I and $\mathrm{CM}$ data sets, respectively. Solid line: $\lambda_{\max }=0.038 R_{V}+0.43 ;$ dotted line: $\lambda_{\max }=0.24 R_{V}-0.27$ which show no effects due to circumstellar dust. Also plotted (as open circles) are the data from Clayton and Mathis (CM) (1988). The CM data set includes stars from both diffuse and dark cloud environments, selected by the removal of all factors not due to the interstellar medium. The scatter in both sets of points is quite high, but leastsquares fits to each show a considerable difference in slopes. In Cha I, there is a larger dispersion in $R_{V}$ than would be expected for its range in $\lambda_{\max }$. This suggests that these parameters are somewhat more independent in Cha I than in other regions, and thus the grains governing these values may well exist independently of one another in different regions of the cloud.

\section{Polarization efficiency}

The value of polarization efficiency (also called "alignment efficiency" by some authors, although it is not only a measure of grain alignment) is defined as the ratio of polarization $(p)$ to extinction (A) or reddening (E) along a single line of sight, and is used as a measure of the rate at which dust grains polarize starlight. It is usually written as $p / A$, and will be used here in units of $\% \mathrm{mag}^{-1}$. The value of $p / A$ will not only be influenced by the grain alignment mechanism, but by several other factors as well, such as the viewing angle with respect to the average magnetic field direction, and the structure and possible multiplicity of interstellar clouds along the line of sight.

\section{1. Chamaeleon $\mathrm{I}$}

The upper bound in diagrams plotting $p$ vs A (or E) represent the optimum polarization efficiency in the sample. In the Serkowski et al. (1975) sample, the upper bound may be expressed in extinction units as $p_{\max } / A_{V}=3 \% \mathrm{mag}^{-1}$, assuming a value of $R_{V}=3$ to convert from reddening to extinction. Figure 2 plots the value of $p_{\max } / A_{V}$ vs $A_{V}$ for the Cha I data set [from Whittet $e t$ al. (1994)]. The upper bound from the Serkowski et al. (1975) sample is shown as a dashed line in this figure, and clearly does not represent the most efficient polarization in Cha I. The solid line shows a more appropriate upper bound for this set, given by $p_{\max } / A_{V}=4.5 \% \mathrm{mag}^{-1}$. Such a high value suggests a lack of depolarization effects in the direction of Cha I, such as the twisting of magnetic field lines or the existence of more than one cloud in the line of sight. A clear trend with $\mathrm{A}_{V}$ is also seen in the data, with polarization efficiency decreasing with increasing extinction. This directly demonstrates that regions with larger extinctions [and higher values of $R_{V}$; see Whittet et al. (1994)] do not provide as large a contribution to polarization as the more diffuse regions. Such a trend also strengthens the argument for the existence of two independent grain populations (mentioned in Section 2)one which exists in the outer regions, providing the main polarization properties of the cloud, and another existing deeper within the cloud, dominating the extinction [as presented by Goodman et al. (1992) for Taurus and Goodman et al. (1995) for the cloud L1755]. 


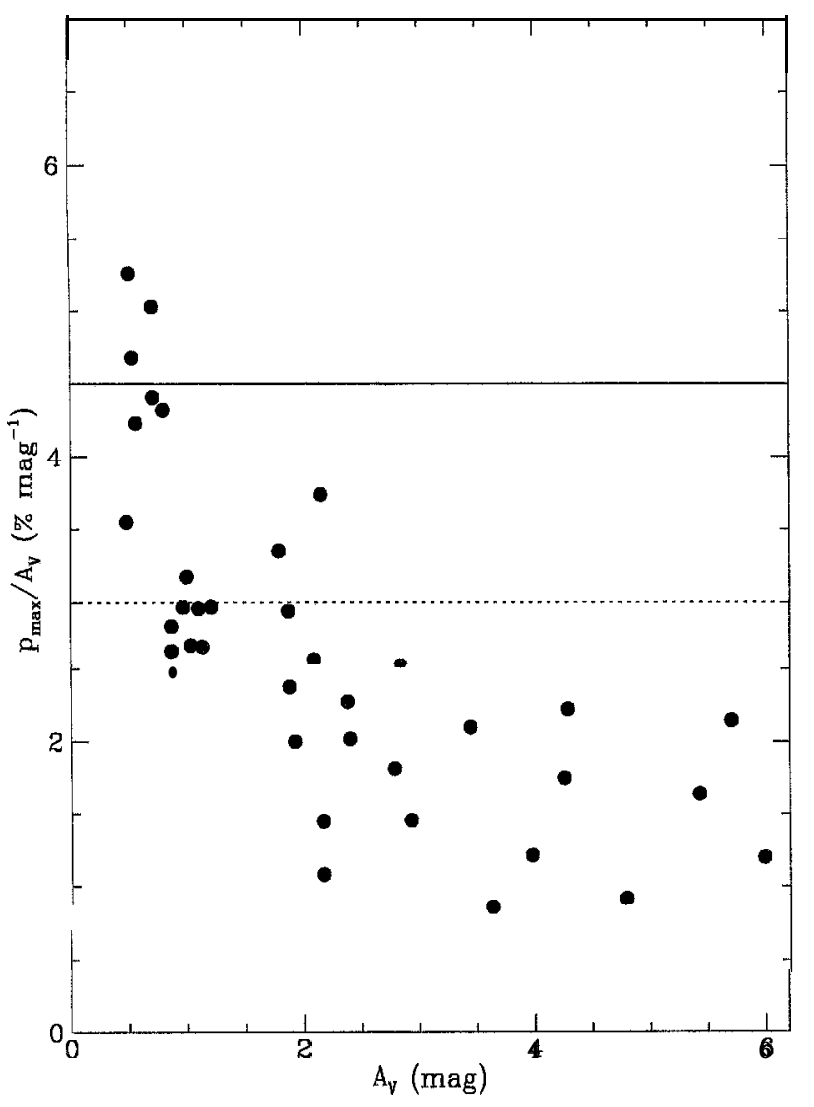

Fig. 2. Plot of polarization efficiency $\left(p_{\max } / A_{V}\right)$ in Cha I. The dashed line represents the upper limit observed in the Serkowski et al. (1975) sample of $p_{\max } / A_{V}=3 \% \mathrm{mag}^{-1}$, and the solid line shows the more appropriate value for Cha I of $p_{\max } / A_{V}=4.5 \%$ $\mathrm{mag}^{-1}$

\subsection{Taurus}

We have studied the polarization efficiency in the Taurus dark cloud by combining observations from four authors, joining them in a single, consistent data set (Gerakines et al., 1995). Specifically, data have been obtained from Moneti et al. (1984), Tamura et al. (1987), Goodman et al. (1992) and Whittet et al. (1992). Values for extinction, where not directly given, were calculated using a standard extinction law $\left(A_{K}=0.57 E_{J_{-K}}\right.$; assuming a spectral type of K3III where unknown). The results of these refinements are plotted in Fig. 3 [from Gerakines et al. (1995)] as polarization efficiency vs extinction in the near-IR $\left(p_{K} / A_{K}\right.$ vs $A_{K}$ ). A dramatic decrease in polarization efficiency is evident for the extinction range covered $\left(A_{K}=0-2.6 \mathrm{mag}\right)$ and has been fit very well with a power law, $p_{K} / A_{K}=$ $1.31 \mathrm{~A}_{K}^{-0.60}$.

Several physical processes may produce such decreases in polarization efficiency, including declining magnetic field strength, kinetic temperature, increases in grain size and icy grain mantle growth. It is interesting to consider this last process in the Taurus dark cloud, since it has been shown by Whittet et al. (1988) that water-ice mantles exist only for visual magnitudes greater than 3.3 mag ( $A_{K} \geqslant 0.33 \mathrm{mag}$ ), where polarization efficiency is lowest.

Since alignment by the suprathermal spin mechanism depends on the surface properties of the grain as well as the abundance of hydrogen atoms, the presence of such

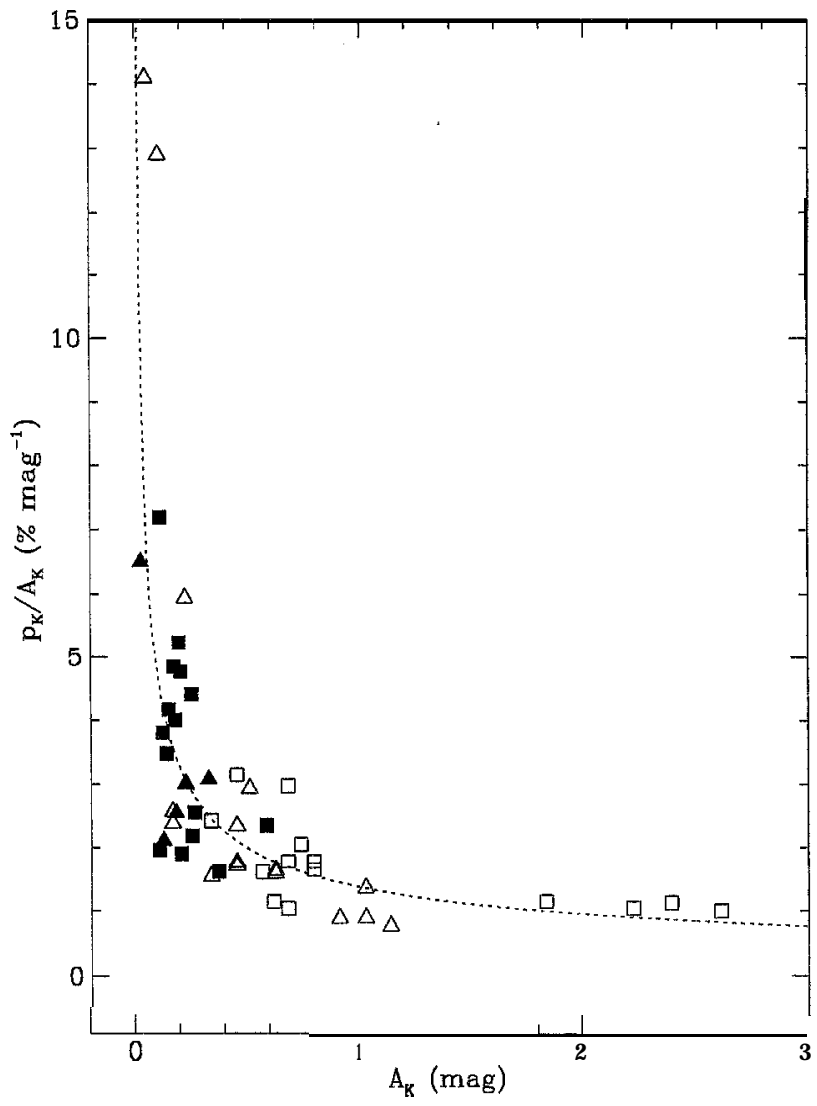

Fig. 3. Plot of K-band polarization efficiency $\left(p_{K} / A_{K}\right)$ vs extinction $\left(A_{K}\right)$ for 40 stars in Taurus. The symbols used indicate the references from which the polarimetry data are taken. Filled squares: Whittet et al. (1992); open squares: Tamura et al. (1987) ; filled triangles: Moneti et al. (1984); open triangles: Goodman et al. (1992). The dotted line represents a least-squares fit to the entire data set, $p_{K} / A_{K}=1.31 A_{K}^{-0.60}$

ice mantles which coat the grain surface could have an observable effect on this process, producing a stronger decline in polarization efficiency with cloud density than expected due to a decline in atomic hydrogen density alone.

\section{Summary and conclusions}

We have examined the dust properties in the Cha I and Taurus dark clouds through investigations of interstellar linear polarization (Whittet et al., 1994; Gerakines et al., 1995). In Cha I, the grain populations responsible for polarization and extinction are found to have a higher degree of independence than toward other regions of the ISM. This cloud also seems to have an atypically higher polarization production efficiency than observed previously for a large number of environments [e.g. Serkowski et al. (1975)]. In Taurus, the polarization efficiency $(p / A)$ has been found to decrease over an order of magnitude as the K-band extinction increases to 2.6 mag. Such a decline may be qualitatively explained through physical processes, although the exact contribution of each is not well known. We propose that icy grain mantle growth in 
dense clouds may largely contribute to this effect, sanceling out a very efficient grain alignment mechanism.

\section{References}

Clayton, G. C. and Matbis, J. S., Astrophys. J. 327, 911 1, 3988.

Coyne, G. V., Gehreis, T. and Serkowski, K., Astron. J. 79, 581, 1974.

Davis, L. and Greenstein, J. L., Astrophys. J. 114, 206, 1951.

Gerakines, P. A., Whittet, D. C. B. and Lazarian, Astrophys. J. Lett. 1995, accepted for publication.

Goodman, A. A., Jones, T. J., Lada, E. A. and Myers P. C., Astrophys. J. 399, 108, 1992.

Goodman, A. A., Jones, T. J., Lada, E. A. and Myers, P. C., Astrophys. J. 1995, submitted.

Heiles, C., in Interstellar Processes (edited by L. J. Allamandola and A. G. G. M. Tielens). Kluwer, Dordrecht, 1987.

Holienbach, D. and Salpeter, E. E., Astrophys. J. 163, 155, 1971.

Jones, R. V. and Spitzer, L., Astrophys. J. 147, 943, 1967.

Mathis, J. S., Astrophys. J. 308, 281, 1986.
Moneti, A., Pipber, J. L., Helfer, H. L., McMillan, R. S. and Perry M. L.,Astrophys. J. 282, 508, 1984.

Purcell, E. M., Astrophys. J. 231,404, 1979.

Serkowski, K., in IAU Symp. 52, Interstellar Dust and Related Topics (edited by J. M. Greenberg and D. S. Hayes). Reidel, Dordrecht, 1973.

Serkowski, K., Methewson, D. S. and Ford, V. L., Astrophys. J. 196,261, 1975.

Tamnra, M., Nagata, T., Sato, S. and Tanaka, M., Mon. Not. R. astron. Soc. 224,413, 1987.

Whittet, D. C. B., Dust in the Galactic Environment. IOP, Bristol, 1992.

Whittet, D. C. B. and van Breda, I. G., Astron. Astrophys. 66, 57, 1978.

Whittet, D. C. B., Bode, M. F., Longmore, A. J., Adamson, A. J., McFadzean, A. D., Aitken, D. K. and Roche, P. F., Mon. Not. R. astron. Soc.233, 321, 1988.

Whittet, D. C. B., Martin, P. G., Hough, J. H., Rouse, M. F., Bailey, J. A. and Axon, D. J., Astrophys. J. 386, 562, 1992.

Whittet, D. C. B., Gerakines, P. A., Carkner, A. L., Hough, J. H., Martin, P. G., Prusti, T. and Kilkenny, D., Mon. Not. R. astron. Soc. 268, 1, 1994. 